\title{
Ketamine induces toxicity in human neurons differentiated from embryonic stem cells via mitochondrial apoptosis pathway
}

\author{
Zeljko J. Bosnjak ${ }^{a, b}$, Yasheng Yan ${ }^{a}$, Scott Canfielda,b, Maria Y. Muravyevaa, Chika Kikuchia, \\ Clive Wells ${ }^{c}$, John Corbett ${ }^{d}$, and Xiaowen Bai ${ }^{a},{ }^{*}$ \\ aDepartment of Anesthesiology, The Medical College of Wisconsin, 8701 Watertown Plank, \\ Milwaukee, WI 53226, USA \\ bDepartment of Physiology, The Medical College of Wisconsin, 8701 Watertown Plank, \\ Milwaukee, WI 53226, USA \\ 'Department of Electron Microscopy Core Facility, The Medical College of Wisconsin, 8701 \\ Watertown Plank, Milwaukee, WI 53226, USA \\ dDepartment of Biochemistry, The Medical College of Wisconsin, 8701 Watertown Plank, \\ Milwaukee, WI 53226, USA
}

\begin{abstract}
Ketamine is widely used for anesthesia in pediatric patients. Growing evidence indicates that ketamine causes neurotoxicity in a variety of developing animal models. Our understanding of anesthesia neurotoxicity in humans is currently limited by difficulties in obtaining neurons and performing developmental toxicity studies in fetal and pediatric populations. It may be possible to overcome these challenges by obtaining neurons from human embryonic stem cells (hESCs) in vitro. hESCs are able to replicate indefinitely and differentiate into every cell type. In this study, we investigated the toxic effect of ketamine on neurons differentiated from hESCs. Two-week-old neurons were treated with different doses and durations of ketamine with or without the reactive oxygen species (ROS) scavenger, Trolox. Cell viability, ultrastructure, mitochondrial membrane potential $\left(\Delta \Psi_{\mathrm{m}}\right)$, cytochrome $\mathrm{c}$ distribution within cells, apoptosis, and ROS production were evaluated. Here we show that ketamine induced ultrastructural abnormalities and dose- and timedependently caused cell death. In addition, ketamine decreased $\Delta \Psi_{\mathrm{m}}$ and increased cytochrome $\mathrm{c}$ release from mitochondria. Ketamine also increased ROS production and induced differential expression of oxidative stress-related genes. Specifically, abnormal ultrastructural and $\Delta \Psi_{\mathrm{m}}$ changes occurred earlier than cell death in the ketamine-induced toxicity process. Furthermore, Trolox significantly decreased ROS generation and attenuated cell death caused by ketamine in a dose-dependent manner. In conclusion, this study illustrates that ketamine time- and dosedependently induces human neurotoxicity via ROS-mediated mitochondrial apoptosis pathway and that these side effects can be prevented by the antioxidant agent Trolox. Thus, hESC-derived neurons might provide a promising tool for studying anesthetic-induced developmental neurotoxicity and prevention strategies.
\end{abstract}

\footnotetext{
"Correspondence to Dr. Xiaowen Bai, Department of Anesthesiology, Medical College of Wisconsin, 8701 Watertown Plank Road, Milwaukee WI, 53226, Phone: 414-456-5755, Fax: 414-456-6122, xibai@ mcw.edu.

Part of this work has been presented at the EXPERIMENTAL BIOLOGY meeting, Washington, April 19, 2011 and at the AMERICAN SOCIETY OF ANESTHESIOLOGIST ANNUAL meeting, Chicago, October 16, 2011.
}

CONFLICT OF INTEREST

The authors have no conflict of interest. 


\section{Keywords}

Embryonic stem cells; neurons; differentiation; neurotoxicity; ketamine

\section{INTRODUCTION}

In the United States millions of infants and young children are exposed annually to various anesthetics. In addition, up to $2 \%$ of pregnant women undergo anesthesia during their pregnancy for surgery unrelated to the delivery. However, growing evidence shows that general anesthetic exposure during the very active brain growth in immature animals results in considerable brain neuronal death and subsequent learning disabilities [1-4]. Ketamine is a noncompetitive N-Methyl-D-aspartate receptor antagonist and widely used in pediatric anesthesia to provide sedation/analgesia to children for painful procedures [5]. In addition, ketamine is one of the most studied anesthetics for addressing neurotoxicity issues in both animal models and animal-derived cell culture system. Neuroapoptosis is one of the commonly recognized mechanisms for the anesthetic-induced developing neurotoxicity. It has been shown that 5 hours of ketamine exposure significantly induced neuroapoptosis in 7-day-old mice [6]. A significant increase in neuroapoptosis was also observed in the cortex of 122 days of gestational and 5-day postnatal rhesus monkeys but not in 35-day postnatal animals [4].

The findings from animal studies raise a real question whether similar neuroapoptosis also occurs in the developing human brain. However, there is considerable controversy about whether rodent and primate data regarding anesthetic-induced neuroapoptosis are clinically relevant. So far, there is no direct evidence that would link exposure to anesthesia or sedative drugs to neurotoxicity. Several retrospective epidemiological studies suggest that anesthesia administration early in life is associated with learning and behavioral abnormalities later in life [7-9]. However, it is clearly very hard to separate the effects of anesthetics alone from other quite relevant variables including the impact of surgery and the factors associated with diseases [10]. In fact, anesthetic neurotoxicity studies in humans are not feasible because it is difficult to obtain neurons and perform developmental toxicity studies in pediatric and fetal populations. No one would consider either avoiding surgery in sick children or exposing healthy children to anesthetic drugs for studying these effects. Since the use of anesthetics cannot be avoided, an understanding of the effects of anesthetics in human neurons is crucial for the safety of fetus, infant, and the young child. It may be possible to overcome these challenges by obtaining developing neurons from human embryonic stem cells (hESCs) in vitro.

hESCs are pluripotent stem cells obtained from the inner cell mass of human embryos at preimplantation stage. hESCs are able to replicate indefinitely and virtually differentiate into every cell type found in the adult body [11]. The differentiation ability of hESCs into committed cell types is potentially valuable for studying cellular and molecular events involved in early human development under physiological and pathological conditions which is almost impossible to perform in humans [12-15]. It has been shown that hESCderived neurons were similar to human neurons at morphological and structural levels [16]. In one recent study, Nash and colleagues used an in vitro hESC-based neurogenesis system to study ethanol-induced early developmental toxicity. They found that ethanol induced a complex mix of phenotypic changes, including inappropriate increase in stem cell proliferation and loss of trophic astrocytes [17]. In the present study, we recapitulated neurogenesis from hESCs in vitro and used the differentiated human neurons to study ketamine-induced neurotoxicity and the underlying mechanisms. We hypothesized that 
ketamine induced neuroapoptosis via reactive oxygen species (ROS)-mediated mitochondrial pathway.

\section{MATERIALS AND METHODS hESC Culture}

Mitotically inactivated mouse embryonic fibroblasts (MEFs) by mitomycin C (Sigma) were used as feeder cells to support the growth and maintenance of hESCs (H1 cell line, WiCell Research Institute Inc.). Inactivated MEFs were plated in $0.1 \%$ gelatin-coated $60 \mathrm{~mm}$ culture Petri dishes containing Dulbecco's modified Eagle's medium (DMEM) supplemented with $10 \%$ fetal bovine serum (Gibco) in a humidified incubator under normoxic condition (20\% $\mathrm{O}_{2} / 5 \% \mathrm{CO}_{2}$ ) at $37^{\circ} \mathrm{C}$. The following day, hESCs were plated on the layer of MEFs with $\mathrm{hESC}$ culture medium and incubated in a hypoxic incubator $\left(4 \% \mathrm{O}_{2} / 5 \% \mathrm{CO}_{2}\right)$. hESC culture medium consisted of DMEM/F12 supplemented with 20\% knock-out serum (Gibco), $1 \%$ non-essential amino acids, $1 \%$ penicillin-streptomycin, $1 \mathrm{mM} \mathrm{L}$-glutamine (Chemicon), 0.1 $\mathrm{mM} \beta$-mercaptoethanol (Sigma), and $4 \mathrm{ng} / \mathrm{mL}$ human recombinant basic fibroblast growth factor (bFGF; Invitrogen). The medium was changed daily. hESCs were passaged every 5-7 days using a mechanical microdissection method. hESCs with passage numbers between 70 and 80 were used in this study.

\section{Neuronal Differentiation of hESCs}

hESCs underwent a four-step progression that includes embryoid body (EB) culture (initiation of differentiation), rosette cell formation, neural stem cell (NSC) expansion, and neuronal differentiation as follows: (1) EB culture. hESCs in the culture were digested using dispase (1.5 unit/mL) (Invitrogen) for $30 \mathrm{~min}$. Digested hESCs were then transferred to $60 \mathrm{~mm}$ ultra-low-attachment dishes (Corning) and cultured in hESC medium without bFGF under normoxic conditions. The medium was changed every day, and the EBs were visible one day after culturing. Four days later, EBs were switched to neural induction medium consisting of DMEM/F12 supplemented with 1\% N2 (Invitrogen), 1\% non-essential amino acids, $5 \mathrm{ng} / \mathrm{mL}$ bFGF, and $1 \mathrm{mg} / \mathrm{mL}$ heparin (Sigma) for 4 days. (2) Rosette formation. EBs were transferred to Growth Factor Reduced BD Matrigel-coated $60 \mathrm{~mm}$ culture dishes and cultured with neural induction medium at day 8 . The medium was changed every other day. EBs attached to the dishes and formed neural tube-like rosettes with radial arrangements of columnar cells within 5 days. (3) NSC expansion. Two days after rosette formation, rosette cells were gently removed with a $5 \mathrm{ml}$ serological pipette to other dishes and cultured with neural expansion medium containing DMEM/F12 supplemented with 1\% N2, 2\% B27 (Invitrogen), 1\% non-essential amino acids, $20 \mathrm{ng} / \mathrm{mL}$ $\mathrm{bFGF}$, and $1 \mathrm{mg} / \mathrm{mL}$ heparin. One day later, rosette cells rolled up to form a round spheres called NSCs. Half of the medium was replaced by fresh medium every other day. NSCs grew as monolayer while cultured in Matrigel-coated dishes. The seeding density of NSCs was $5 \times 10^{5 / 60} \mathrm{~mm}$ dish. NSCs were passaged every $5-6$ days by digestion with accutase (Innovative Cell Technology). NSCs at passages 4 to 12 were used for neuronal differentiation. (4) Neuronal differentiation. Half a million NSCs were cultured in $60 \mathrm{~mm}$ Matrigel-coated dishes with neuronal differentiation medium consisting of Neurobasal, 2\% B27 (Invitrogen), $0.1 \mu \mathrm{M}$ cyclic adenosine monophosphate, $100 \mathrm{ng} / \mathrm{mL}$ ascorbic acid (Sigma), $10 \mathrm{ng} / \mathrm{mL}$ brain-derived neurotrophic factor, $10 \mathrm{ng} / \mathrm{mL}$ glial cell-derived neurotrophic factor, and $10 \mathrm{ng} / \mathrm{mL}$ insulin-like growth factor 1 (Pepro Tech Inc.). The medium was changed every other day for 2 weeks. Two-week-old neurons differentiated from three independently prepared NSCs (passages 5 to 12 ) were used in this study. 


\section{Immunofluorescence Staining}

Cells cultured on matrigel-coated glass coverslips were fixed with $1 \%$ paraformaldehyde for $30 \mathrm{~min}$. Cells were then washed three times with phosphate buffered saline (PBS) alone or PBS containing $0.5 \%$ Triton X-100 (Sigma) and blocked with 10\% donkey serum for 30 min at room temperature followed by incubation with primary antibodies in a moist chamber for 1 hour at $37^{\circ} \mathrm{C}$. The primary antibodies were mouse anti-Sox 2 (Millipore), microtubuleassociated protein 2 (MAP2) (abcam) and cytochrome c (BD Pharmingen), rabbit antinestin, synapsin I and cleaved caspase-3 (Millipore). After three wash, cells were incubated with an Alexa Fluor 485 donkey anti-mouse IgG and/or with an Alexa Fluor 594 donkey anti-rabbit IgG (Invitrogen) for 1 hour at room temperature. Cell nuclei were stained with TO-PRO®-3 (Invitrogen). The coverslips were then mounted onto the slides and the fluorescence images were recorded using a laser-scanning confocal microscope (Nikon Eclipse TE2000-U). Neuron differentiation efficiency was determined by calculating the percentage of MAP2-positive cells over the total cells stained with TO-PRO®-3. Results were obtained from at least 100 cells in each of three independently differentiated neuron samples.

\section{Ketamine Treatment}

NSCs were cultured in either $60 \mathrm{~mm}$ dishes $\left(5 \times 10^{5}\right.$ cells/dish) or 96 -well tissue culture plates $\left(1 \times 10^{4}\right.$ cells/well $)$ with neuron differentiation medium. Two weeks later, neurons were incubated with different doses of ketamine (Phoenix Pharmaceutical, Inc.) with or without ROS inhibitor Trolox. It was reported that peak blood levels of ketamine were as high as $108 \mu \mathrm{M}$ [18]. Ketamine levels required to maintain anesthesia were approximately 10-20 $\mu \mathrm{M}$ [19]. In addition, experimental evidence from in vitro cell culture and in vivo animal studies demonstrated that ketamine could induce neurotoxicity when administered at high doses and/or for prolonged periods [20-24]. Thus, in this study we treated neurons with a wide range of ketamine concentrations $(20-4000 \mu \mathrm{M})$ for 6,12 , or 24 hours. Cell viability (LDH and MTT assays), apoptosis analysis (cleaved caspase-3 staining, quantification of caspase- 3 activity, TUNEL staining and cytochrome c release), and ROS measurement were performed immediately after ketamine exposure for indicated durations (6, 12, or 24 hours).

\section{MTT Assay}

3-(4,5-dimethyithiazol-2-yl)-2,5-diphenyl-tetrazolium bromide (MTT) assay was used to determine cell viability using a Cell Proliferation Kit I (Roche) by measuring mitochondrial dehydrogenase activity that reduces MTT dye to a colored product. Thus, the extent of MTT metabolism is an indicator of mitochondrial function and cell viability. Neurons were cultured in 96-well plate with $100 \mu \mathrm{L}$ neuronal differentiation medium. Four hours after adding $10 \mu \mathrm{L}$ of the MTT solution to each well, MTT reduction product, formazan, was dissolved in SDS solution at $37^{\circ} \mathrm{C}$ overnight. The absorbance of the purple formazan product was then determined spectrophotometrically at $580 \mathrm{~nm}$ with a reference wavelength of $650 \mathrm{~nm}$ using a microtiter plate reader. The amount of MTT reduction product (representing cell viability) in the ketamine-treated neurons was expressed as \% of notreatment control.

\section{LDH Release Assay}

Lactate dehydrogenase (LDH) release into the culture media is an index of cell membrane damage. LDH activity was analyzed using a LDH assay kit (Roche) following the manufacturer's protocol. Briefly, NSCs differentiated from hESCs were cultured in 96-well culture plates with $100 \mu \mathrm{L}$ neuronal differentiation medium. The medium was changed every two days. Two weeks later, $100 \mu \mathrm{L}$ of substrate mix was added to each well in the plates and incubated for $20 \mathrm{~min}$ at room temperature. The reaction was terminated with a 
stop solution and the sample was quantified spectrophotometrically at $490 \mathrm{~nm}$ (reference wavelength is $600 \mathrm{~nm}$ ) with a Microplate Reader (Bio Tek). LDH release from ketaminetreated neurons was presented as fold increase over no-treatment control.

\section{Caspase-3 Activity Quantification}

Caspase-3 activity was measured using Caspase-3 Colorimetric Assay Kit (Genescript) following the manufacturer's protocol. Neurons cultured in $60 \mathrm{~mm}$ dishes were washed twice with PBS and lysed with $50 \mu \mathrm{L}$ lysis buffer followed by adding $50 \mu \mathrm{L}$ reaction buffer as well as $5 \mu \mathrm{L}$ substrate. After 4-hour incubation at $37^{\circ} \mathrm{C}$, caspase- 3 activity was measured using a Microplate Reader at $400 \mathrm{~nm}$. The values of optical density at $400 \mathrm{~nm}$ was normalized to total protein content of the samples as determined by DC Protein Assay Reagents Package Kit (Bio-Rad).

\section{TUNEL Assay}

DNA fragmentation associated with apoptosis was analyzed by performing terminal deoxynucleotidyl transferase-mediated deoxyuridine triphosphate in situ nick end labeling (TUNEL) staining using an in situ apoptotic cell death detection kit (Roche Applied Bio Sciences) following the manufacturer's instruction. The cells cultured on coverslips were rinsed with PBS, fixed with ice-cold $1 \%$ paraformaldehyde. Terminal deoxynucleotidyl transferase, a template-independent polymerase, was used to incorporate nucleotides at the sites of DNA breaks. Nuclei were stained with TO-PRO®-3, and the fluorescent images were taken at three different fields for each coverslip using the confocol microscope. Apoptotic index was calculated as a percentage according to the following formula: TUNEL-positive nuclei number/the number of total cell nuclei.

\section{Electron Microscopy-Ultrastructural Analysis}

Neurons cultured on matrigel-coated coverslips from each experimental condition were fixed with $2 \%$ glutaraldehyde in $0.1 \mathrm{M}$ sodium cacodylate buffer at $4^{\circ} \mathrm{C}$, washed in the same buffer, and post-fixed with $1 \%$ osmium tetroxide for $60 \mathrm{~min}$ on ice. Cells were then washed briefly in distilled water and dehydrated through graded methanol (50\%, $20 \mathrm{~min} ; 70 \%, 20$ $\min ; 95 \%, 20 \mathrm{~min} ; 100 \%, 3 \times 20 \mathrm{~min})$ and acetonitrile $(2 \times 10 \mathrm{~min})$. The cells were infiltrated with epoxy resin (EMbed-812; Electron Microscopy Sciences, Hatfield, PA), and polymerized overnight at $70^{\circ} \mathrm{C}$. Ultra-thin sections $(\sim 60 \mathrm{~nm})$ were cut, stained with uranyl acetate and lead citrate according to standard procedures and viewed with a Hitachi H600 EM.

\section{Mitochondrial Membrane Potential $\left(\Delta \boldsymbol{\Psi}_{\mathrm{m}}\right)$ Assay}

Tetramethylrhodamine ethyl ester (TMRE) is a lipophilic, positively charged dye. TMRE can penetrate intact live cells and enter the negatively charged mitochondria where it accumulates in an inner-membrane potential-dependent manner. When the $\Delta \Psi_{\mathrm{m}}$ collapses in apoptotic cells, TMRE no longer accumulates inside the mitochondria and becomes more evenly distributed throughout the cytosol. When dispersed in this manner, overall cellular fluorescence levels drop dramatically and this event can easily be visualized by fluorescence microscopy. Neurons cultured on glass coverslips were loaded with $50 \mathrm{nM}$ TMRE (Invitrogen) for $20 \mathrm{~min}$ at room temperature. TMRE fluorescence intensity representing $\Delta \Psi_{\mathrm{m}}$ was recorded with the confocol microscope and analyzed by ImageJ software 1.41 (Wayne Rasband). Results were obtained from at least 100 cells in each of three samples per group. 


\section{Labeling Mitochondria and Characterization of Distribution of Cytochrome $\mathbf{C}$ in Neurons}

To indentify if ketamine induces cytochrome c release from mitochondria into cytosol, we labeled mitochondria as described as follows. Ten days after NSCs were cultured in neuronal differentiation medium, differentiated neurons were transduced with the virus CellLight ${ }^{\mathrm{TM}}$ mitochondria-GFP (green fluorescence protein) (Invitrogen) to label the mitochondria following the instructions provided by the company. This fluorescent proteinbased reagent contains the leader sequence of E1alpha pyruvate dehydrogenase fused to emerald GFP. Transduced neurons expressed GFP within mitochondria. Four days later, the labeled neurons were used for the analysis of the effect of ketamine on the cytochrome $\mathrm{c}$ translocation. The distribution of cytochrome $\mathrm{c}$ in the neurons expressing GFP within mitochondria was analyzed with antibody against cytochrome c (BD Pharmingen) using immunofluorescence staining.

\section{ROS Measurement}

The intracellular ROS level in neurons was detected by labeling neurons with 5-(and-6)chloromethyl-2' $7^{\prime}$-dichlorodihydrofluorescein diacetate, acetyl ester (CM- $\left.\mathrm{H}_{2} \mathrm{DCFDA}\right)$ (Invitrogen), a ROS-sensitive membrane permeable fluorescent probe. Upon penetration into the cells, the acetate groups of CM- $\mathrm{H}_{2} \mathrm{DCFDA}$ are cleaved by cytoplasmic esterases to form membrane impermeable non-fluorescent $\mathrm{CM}-\mathrm{H}_{2} \mathrm{DCF}$ trapped within the cells. $\mathrm{CM}-\mathrm{H}_{2} \mathrm{DCF}$ is then rapidly oxidized by ROS to produce highly fluorescent dye DCF. DCF fluorescent intensity represents ROS production. The neuron-contained coverslips were placed in a polycarbonate recording chamber (Warner Instruments) on the stage of the confocal microscope with the $\times 60 / 1.4$ oil-immersion objective. Neurons were loaded with $10 \mu \mathrm{M}$ CM- $\mathrm{H}_{2}$ DCFDA for $30 \mathrm{~min}$ in the presence/absence of ketamine with or without Trolox followed by 20-min washout. The DCF fluorescence of cells was acquired with the confocol microscope at excitation (by argon laser) and emission wavelengths: $\lambda_{\mathrm{ex}} / \lambda_{\mathrm{em}}=488 / 500-550$ within 8 min. DCF intensity was quantified with ImageJ software 1.41 (Wayne Rasband). Results were obtained from at least 100 neurons in each of five experiments per group.

\section{Real-Time PCR Analysis}

Total RNA was extracted from cells using RNAqueous-micro scale RNA isolation kit (Ambion) and was reverse transcribed to cDNA using iScriptTM cDNA systhesis kit (BioRad). cDNA was then subjected to PCR amplification using $\mathrm{iQ}^{\mathrm{TM}}$ SYBR green supermix (Bio-Rad) in a final volume of $25 \mu \mathrm{l}$ with each specific primer set using the iCycler iQ Detection System (Bio-Rad Laboratories). PCR reaction cycles were programmed as follows: $95^{\circ} \mathrm{C}$ for $3 \mathrm{~min}, 50$ cycles of $10 \mathrm{~s}$ denaturation at $95^{\circ} \mathrm{C}, 60 \mathrm{~s}$ at $60^{\circ} \mathrm{C}$. The threshold cycle $(\mathrm{Ct})$ of each well was determined. The relative expression of each mRNA was calculated by the comparative $\mathrm{Ct}$ method and normalized to the housekeeping gene, beta-2microglobulin, using the formula: $\Delta \mathrm{Ct}=\mathrm{Ct}$ of the gene of interest $-\mathrm{Ct}$ of beta-2microglobulin. The fold change of the individual gene expression was presented by the $2^{-\Delta \Delta \mathrm{Ct}}$ formula in which $\Delta \Delta \mathrm{Ct}=\Delta \mathrm{Ct}$ of ketamine group $-\Delta \mathrm{Ct}$ of no-treatment group. The following oxidative stress-related genes were analyzed: glutathione synthetase (GSS), glutathione reductase (GSR), oxidation resistance 1 (OXR1), oxidative-stress responsive 1 (OXSR1), superoxide dismutase 1 (SOD1), and glutathione peroxidase 1 (GPX1). The primers used were purchased from RealTimePrimers.COM.

\section{Statistical Analysis}

Reported values were expressed as means \pm standard deviation from results obtained from three independent neuronal differentiations. The statistically significant differences between groups were tested by using one-way analysis of variance or Student's t-test using SPSS software version 15 . A level of $\mathrm{P} \unlhd \mathbf{} \mathbf{0} .05$ was considered to be statistically significant. 


\section{RESULTS}

\section{Differentiation of hESCs into Neurons}

$\mathrm{hESCs}$ were induced to differentiate into neurons in vitro via NSC step. NSCs were confirmed by the expression of NSC-specific markers Sox 2 and nestin (Fig. 1A). NSCs showed strong proliferation potential. They were passaged every 5-6 days for over 12 passages. Obvious neuronal differentiation could be observed in the culture 3 days after NSCs were cultured in neuronal differentiation medium. Differentiated neurons formed extensive neuron networks over time. Two-week-old cultured neurons expressed neuronspecific marker MAP2. Over $90 \%$ of the NSCs differentiated into MAP2-positive neurons within 2 weeks of protocol initiation. In addition to MAP2, differentiated neurons were also positive for synapsin I, the marker used to define the presence of presynaptic vesicles [25]. Puncta-like synapsin 1 could be observed around cell bodies and along radially oriented axons (Fig. 1B). These hESC-derived cells were similar to human neurons at morphological and structural levels as previously described [16].

\section{Ketamine Time- and Dose-Dependently Decreased Neuronal Viability}

We next investigated whether ketamine caused neuronal death. Two-week-old neurons were treated with increasing concentrations of ketamine $(20-4000 \mu \mathrm{M})$ for different durations. Lower dose ketamine (20 to $2000 \mu \mathrm{M}$ ) treatment for 24 hours did not influence the overall cellular morphology. However, $2500 \mu \mathrm{M}$ ketamine caused cellular projection retraction and cell detachment. Most cells were detached in the $4000 \mu \mathrm{M}$ ketamine-treated culture (Fig. $2 \mathrm{~A}$ ). We then used two methods to analyze cell viability: MTT and LDH assays. Using MTT assay, we found that at the concentrations up to $2000 \mu \mathrm{M}$, ketamine did not modify cell viability. However, $3500 \mu \mathrm{M}$ ketamine treatment for 6 hours significantly decreased cell viability $(\mathrm{P}<0.01, \mathrm{n}=3)$. In addition, higher concentrations and longer treatment durations induced more cell death. Cell viability was $92 \pm 2 \%, 72 \pm 5 \%$, and $59 \pm 2 \%$ of the notreatment control after 6,12 , or 24 hours of incubation with $3000 \mu \mathrm{M}$ ketamine $(\mathrm{P}<0.01$, $\mathrm{n}=3$ ) (Fig. 2B). In accordance with this loss of cell viability (MTT assay), higher concentrations and longer exposures to ketamine resulted in the enhanced LDH release in the culture. $\mathrm{LDH}$ release in the $4000 \mu \mathrm{M}$ ketamine-treated cells for 24 hours was 1.57 -fold \pm 0.06 higher than in no-treatment culture (Fig. 2C). Collectively, these combined data showed that ketamine dose- and time- dependently caused neuronal death.

\section{Ketamine Leads to Neuronal Ultrastructural Abnormalities}

We next investigated if ketamine caused ultrastructural change in the neurons using electron microscope. Figure 3A shows the representative images of neurons treated with various concentrations of ketamine for 24 hours. Normal neurons had very elongated mitochondria with varying lengths of up to and over $4 \mu \mathrm{m}$. Other organelles such as Golgi apparatus and rough endoplasmic reticulum (ER) appeared regular. The cytoskeleton (filaments and tubules) of the cells was regularly ordered and appeared in numerous parallel arrays along the length of the cells. However, ketamine caused abnormal ultrastructure in neurons. Specifically, as shown in Figure 2, the lower concentrations (200 and $1000 \mu \mathrm{M})$ of ketamine-treated cultures did not induce cell death. However, Figure 3A shows the representative electron microscope images indicating a clear ultrastructural change in these low-dose ketamine-treated cultures. The detailed descriptions of ultrastructural abnormalities in the ketamine-treated neurons are as follows: (1) In the $200 \mu \mathrm{M}$ ketaminetreated culture, the length of mitochondria was short indicating mitochondrial fragmentation. Golgi structures were not observed. Autophagosomes were very pronounced and found in almost every cell often occupying the majority of cytosol volume. (2) Neurons treated with $1000 \mu \mathrm{M}$ ketamine showed signs of traumatic damage. Mitochondria were much shorter (1$1.5 \mu \mathrm{m}$ long) than in the controls. The cristae appeared somewhat irregular and disrupted. 
The Golgi apparatus were scarce and difficult to identify as they appeared to be losing cohesion and were much more vesicular with few intact stacks seen. The rough ER was becoming less abundant and distended with fewer and less contrasted ribosomes along its length. The nuclei were still regular oval in shape with sparse dense chromatin and pronounced nucleoli. There appeared to be numerous large autophagosomes measuring between 1-2 $\mu \mathrm{m}$ within the majority of cells. These were packed with whorls of membranelike material and dense deposits. The cytoskeletal fibers and tubules were still visible but appeared much more disordered than the control cells and rarely showing parallel arrays. (3) In the $3000 \mu \mathrm{M}$ ketamine-treated culture, mitochondria were spherical or oval and sparse with few cristae visible. No Golgi structures were observed. Somewhat diminished ER that was seen was distended with few ribosomes along its length. The nuclei were small, sometimes pyknotic, irregularly shaped with prominent chromatin and nucleoli. There were many autophagosomes packed with dense amorphous material and other large clear vacuoles within the cytosol of all cells. A few highly disorganized cytoskeletal filaments were observed and no tubules were present.

\section{Ketamine Dose- Dependently Causes Depolarization of Mitochondrial Membrane Potential of Neurons}

Given that ketamine may cause mitochondrial damage, we then measured $\Delta \Psi_{\mathrm{m}}$. Figure 3B shows the confocol images of the neurons loaded with mitochondrial probe TMRE. The fluorescent intensity representing $\Delta \Psi_{\mathrm{m}}$ was significantly decreased in the cultures following 24 hours of ketamine treatment. Ketamine-induced decrease in $\Delta \Psi_{\mathrm{m}}$ displayed dose dependency ( $\mathrm{P}<0.01 \mathrm{vs}$. control). $\Delta \Psi_{\mathrm{m}}$ in control, $200 \mu \mathrm{M}, 1000 \mu \mathrm{M}$ and $3000 \mu \mathrm{M}$ ketamine-treated cultures were $1960.85 \pm 83.77,1382.94 \pm 36.75,671.49 \pm 153.95$ and $126.15 \pm 62.37$, respectively (Fig. 3C).

\section{Ketamine Induces Neuronal Toxicity via Apoptotic Pathway}

In order to investigate whether the observed ketamine-induced neurotoxicity may be related to occurrences of apoptosis, we measured cleavage caspase-3 activity and examined DNA damage. Figure 4A demonstrates that $3000 \mu \mathrm{M}$ ketamine treatment for 24 hours significantly increased the number of cells that stained positive for cleaved caspase- 3 . Quantification of the activity of cleaved caspase-3 further showed that there was higher caspase-3 activity in the ketamine-treated culture than in no-treatment group $(0.50 \pm 0.01$ vs. $0.28 \pm 0.02)(\mathrm{P}<0.01, \mathrm{n}=3)$ (Fig. 4B). Consistent with caspase-3 cleavage and activation, numerous darkly stained TUNEL-positive cells were observed in ketamine-treated cultures (Fig. 4C). Most cells stained positive for TUNEL were located in condensed nuclei resulted from chromatin condensation, a hallmark of apoptosis. Ketamine significantly induced an increase in TUNEL-positive cells (30.98 $\pm 6.91 \%$ in ketamine-treated culture and $11.65 \pm$ $0.66 \%$ in control culture, respectively) $(\mathrm{P}<0.01, \mathrm{n}=3)$ (Fig. 4D).

\section{Ketamine Causes Cytochrome C Release from Mitochondria into Cytosol}

In mammalian cells, a major caspase activation pathway is the cytochrome c-initiated pathway. In this pathway, cytochrome c releases from mitochondria into cytosol, which in turn induces a series of biochemical reactions that result in apoptosis. As shown in Figure $4 \mathrm{E}$, treatment of neurons with $3000 \mu \mathrm{M}$ ketamine for 24 hours facilitated cytochrome c release from the mitochondria into cytosol as compared with the control condition.

\section{Ketamine Induces ROS Production in Neurons}

ROS production was determined by DCF fluorescence intensity in the neurons treated with or without ketamine. Figure 5A shows representative images of the DCF fluorescence in neurons. The relatively faint DCF signals were observed in control neurons, while DCF 
fluorescence intensity was increased in the cultured treated with $3000 \mu \mathrm{M}$ ketamine for 24 hours. Ketamine stimulated 2.52-fold increase in the production of ROS in neurons compared with control ( $258.72 \pm 100.72$ vs. $102.78 \pm 19.49)$ (Fig. 5B).

\section{Ketamine Induces Differential Regulation of Oxidative Stress-Related Genes}

We examined the expression of six oxidative stress-related genes (GSS, GSR 1, OXR1, OXSR, SOD1, and GPX1) in neurons using real-time PCR. GSS, OXR1, and OXSR1 are involved in oxidative stress. GSR and GPX1 are involved in peroxidase activity and oxidoreductase activity. SOD1 is involved in superoxide metabolism and oxidoreductase activity. These six oxidative stress-related genes were found to be differentially regulated with ketamine treatment. There were up-regulations of GSS (1.89-fold), GSR (9.69-fold), OXR1 (11.13-fold), OXSR1 (5.31-fold), and SOD1 (14.02-fold). There was a downregulation of GPX1 (0.55-fold) (Fig. 5C).

\section{ROS Scavenger Trolox Protects Neurons against Ketamine-Induced Neurotoxicity}

To determine if ROS production mediates the cleavage of capsase- 3 and induces neuronal death, differentiated neurons were treated with ketamine with or without Trolox. As expected, Trolox $(250 \mu \mathrm{M})$ had a significant inhibitory effect on ROS generation in the $3000 \mu \mathrm{M}$ ketamine-treated neurons for 24 hours (2.52-fold \pm 0.98 in ketamine alone group vs. 1.02-fold \pm 0.26 in ketamine plus Trolox group, $n=3$ ) (Figs. 6A and B). This inhibition of ROS was associated with an attenuation in ketamine-induced caspase- 3 activation (1.77-fold \pm 0.05 in ketamine group vs. 1.36 -fold \pm 0.19 in ketamine plus Trolox group $(\mathrm{P}<0.05, \mathrm{n}=3)$ (Figs. 6C and D). We then determined if Trolox completely reversed ketamine-induced cell death. Neurons were treated with different concentrations of Trolox (range $0-1000 \mu \mathrm{M}$ ). Following 24-hour incubation with ketamine, there was a $41 \%$ decrease in neuronal viability. Trolox $(250 \mu \mathrm{M})$ significantly increased cell viability to $75 \%$. In addition, Trolox exhibited concentration-related protective effects. Ketamine-induced cell death was completely attenuated by $1000 \mu \mathrm{M}$ Trolox (Cell viability: $59.27 \pm 2.30 \%$ of control in ketamine group vs. $74.75 \pm 6.00 \%$ in ketamine $+250 \mu \mathrm{M}$ Trolox and $103.56 \pm 6.50 \%$ in ketamine $+1000 \mu \mathrm{M}$ Trolox) (Figs. $6 \mathrm{E}$ and F).

\section{DISCUSSION}

In this study, we investigated the toxic effect of ketamine on the human neurons differentiated from hESCs. Our major findings are summarized as follows: (1) Ketamine decreased neuronal viability in a concentration- and time-dependent manner; (2) Ketamine increased caspase-3 activity and DNA damage, decreased $\Delta \Psi_{\mathrm{m}}$ and induced cytochrome c release from mitochondria into cytosol, indicating that ketamine-treated neurons undergo mitotochondria-mediated apoptosis; (3) Ketamine significantly induced ultrastructural abnormalities in neurons, including mitochondrial fragmentation, a dramatic decrease in Golgi and rough ER, and an increase in autophagosomes; (4) Ketamine-treated neurons generated higher levels of ROS than control cells; and (5) Trolox, a ROS scavenger, significantly attenuated ketamine-induced ROS formation, the increased caspase- 3 activity, and cell death.

To study ketamine-induced neurotoxicity we mimicked developmental principles to obtain hESC-derived neurons that were similar to human neurons at morphological and structural levels (Fig. 1B). These differentiated neurons were reported to generate action potentials and induce the synaptic transmission [16]. We then used multiple methods to analyze multiparametric indicators of cellular toxicity inferring mechanisms of cell death and covering a wide spectrum of cytopathological changes including cell viability, apoptosis, mitochondrial dysfunction, ultrastructural pathology and ROS production. The data from MTT and LDH 
assays revealed that ketamine at concentrations lower than $2000 \mu \mathrm{M}$ did not induce cell death even after prolonged exposures ( 24 hours). A significant decrease in neuronal viability was observed only when ketamine concentration was raised to greater than $2000 \mu \mathrm{M}$. These results were largely in line with several recent studies by others who also found that ketamine-induced neurotoxicity in vitro occurred after prolonged exposures with high concentrations. For instance, Mak et al. demonstrated that administration of $4000 \mu \mathrm{M}$ ketamine for 48 hours caused significant death of differentiated neurons from human $\mathrm{SH}-$ SYY5 neuroblastoma cell line [22]. This finding was supported by Braun et al. who showed a significant increase in the number of apoptotic neurons differentiated from human SHEP neuroblastoma cell line after 24 hours of $2000 \mu \mathrm{M}$ ketamine treatment [20]. A similar finding was also reported in primary cortical neurons harvested from postnatal day 2-8 mice. In this study, clinically relevant anesthetic concentrations of ketamine had no significant neurotoxic effects, and only reaching significant levels of neurotoxicity when the ketamine concentration was raised to above clinically applied concentrations (e.g., 1-3 mM ketamine) [21]. In contrast, some studies showed the toxic effect of comparatively low concentrations of ketamine on cultured neurons. For instance, treatment of fetal rat neurons after 18-19 days gestation with $100 \mu \mathrm{M}$ of ketamine for 48 hours resulted in the loss of $45 \%$ of neurons by apoptosis [26].

The reasons why different studies showed various toxic thresholds of ketamine-induced neuronal death may be explained as follows. First, the sensitivity of neurons obtained from different species to ketamine may differ. Second, neurons used in different studies might be at different developmental stages or contain different percentage of population of neurons influencing the vulnerability to ketamine exposure. It is commonly considered that developing mammals are at greatest apoptotic risk during the most rapid period of growth of their central nervous system. In rats, brain growth peaks at approximately 7 days after birth. For rhesus monkeys the peak growth takes place after approximately 115 days of gestation [27] [28]. In humans, neurogenesis is the most active during first trimester of pregnancy [29] while synaptogenesis starts during the third trimester and continues for up to 2 to 3 years [30]. Thus, neurons at different stages of brain growth burst might also exhibit different sensitivity to ketamine. Third, in vitro neuronal culture system excludes the influence of other environmental factors in vivo that may increase toxic threshold of ketamine. Last, neuron death may be the severe side effect caused only by high doses of ketamine. In our current study, our criteria for neurotoxicity was a significant decrease in the cell viability analyzed by MTT and LDH assay. Thus, we cannot rule out the possibility that neurons were compromised by clinically relevant dose of ketamine exposure and this impact was not detected by our assays.

An increase in LDH release and a decrease in MTT metabolism resulted from $3000 \mu \mathrm{M}$ ketamine treatment only suggest the damage of cell membrane and impaired mitochondrial function (Fig. 2). Thus, we hypothesized that low concentration of ketamine might also exert other harmful effects in addition to cell death. We then used electron microscope to study the ketamine-induced toxic effect. Specifically, we found for the first time that lower doses of ketamine $(200$ and $1000 \mu \mathrm{M})$ led to significant ultrastructural abnormalities (including mitochondrial fragmentation, a decrease in Golgi and rough ER, and an increase in autophagosomes) (Fig. 3A). $\Delta \Psi \mathrm{m}$ was also significantly decreased in the $200 \mu \mathrm{M}$ ketamine-treated culture (Fig. 3B and C). These data indicate that abnormal ultrastructure and $\Delta \Psi \mathrm{m}$ are earlier indicators for ketamine-induced neurotoxicity compared with the decrease in cell viability. Ketamine might also alter other cell physiological activities, such as neuronal receptor expression, structure and branching of neurons' dendrites and synaptogenesis, eventually resulting in impaired neuronal function. 
It is widely accepted that general anesthetics cause neuronal loss by the inappropriate activation of apoptotic cell death pathways [20, 26, 31, 32]. Apoptosis plays a very important role in development, aging and disease such as neurological disorders (e.g., Alzheimer's disease) [33]. Apoptosis is mediated by two central pathways involving either the intrinsic (or mitochondrial) pathway or the activation of the extrinsic (or death receptors) pathway. Both pathways converge with the activation of caspases followed by the cleavage of specific substrates, leading to the cell death. The mitochondrial pathway of apoptosis begins with the permeabilization of the mitochondrial outer membrane. The mechanisms through which this occurs remain controversial. However, it is thought that loss of $\Delta \Psi_{\mathrm{m}}$ and release of cytochrome $\mathrm{c}$ from the mitochondria are key events in initiating mitochondriainvolved apoptosis $[34,35]$. The released cytochrome $\mathrm{c}$ in the cytosol activates caspase-9, which consequently induces caspase- 3 activation, resulting in the cleavage of several cellular proteins, finally leading to the typical alterations related to cell apoptosis (e.g., DNA fragmentation in cell nuclei) $[20,36]$. In this study, following ketamine exposure, there was a significant increase in the caspase-3 activity as well as TUNEL-positive cells with condensed and fragmented nuclei (Fig. 4A to D). In addition, ketamine-induced neuronal apoptosis was accompanied by a significant decrease in $\Delta \Psi_{\mathrm{m}}$ (Fig. 3C) and the release of cytochrome $\mathrm{c}$ from mitochondria into cytosol (Fig. 4E), suggesting that ketamine induces human neurons to undergo mitochondria-mediated apoptosis pathway.

The mechanistic details by which anesthetics induce or trigger neuroapoptosis have yet to be established. We hypothesized that ketamine-induced ROS formation mediated the toxicity in the differentiated neurons. Several metabolic pathways, including the mitochondrial electron transport chain, are known to produce ROS [37]. Oxidative stress resulted from the cellular accumulation of ROS has been shown to be important in the execution of neuronal apoptosis, contributing to the pathogenesis of neurodegeneration in various disease such as Alzheimer's and Parkinson's disease [38]. One recent study demonstrated that ROS production was increased following the exposure of mouse neurons isolated from mouse at gestation stage of day 15 to volatile anesthetic isoflurane [36]. In this present study, in response to ketamine, there was a significant increase in ROS production (2.4-fold of control) in the neurons. In addition, ketamine induced differential regulation of oxidative stress-related gene expression. There was a significant up-regulation of antioxidant enzymes (GSR and SOD1) for elimination of ROS and the enzymes (GSS, OXR1, and OXSR1) that are involved in oxidative stress (Fig. 5). Importantly, Trolox, a ROS scavenger, not only attenuated ketamine-induced ROS production, but also decreased caspase- 3 activation and prevented cell death (Fig. 6), suggesting that ketamine-induced neuroapoptosis is directly associated with enhanced ROS production.

Mitochondria are highly dynamic organelles that undergo continual fusion and fission to form networks which are controlled by multiple proteins including dynamin-related GTPase protein 1 (Drp1). Unbalanced fission-fusion could affect a variety of biological processes such as apoptosis, leading to various pathological processes including neurodegeneration [39-41]. Increased mitochondrial fission in apoptotic cells apparently paralleled the release of cytochrome $\mathrm{c}$ while inhibition of fission by Drp1-RNA interference delayed the release of cytochrome c [42]. Increased mitochondrial ROS-mediated mitochondrial fission was reported to be followed by cellular dysfunction [43]. As we showed in Figure 3A, ketamine also induced mitochondrial fission in the neurons which might be involved in ketamineinduced neuroapoptosis.

\section{CONCLUSION}

We utilized differentiated neurons from stem cells to study ketamine-induced human neurotoxicity by analyzing multi-parametric indicators of cellular toxicity including cell 
viability, apoptosis, mitochondrial dysfunction, ultrastructural pathology and ROS production. The results demonstrate that (1) ketamine induces toxicity in human neurons via ROS-involved mitochondria-mediated apoptosis pathway in a dose- and time-dependent fashion; (2) abnormal ultrastructural and $\Delta \Psi_{\mathrm{m}}$ changes occur earlier than cell death in the ketamine-induced toxicity process; (3) this harmful effect could be prevented by the ROS scavenger Trolox; and (4) neurons differentiated from hESCs might be a promising tool for studying neurotoxicity caused by various anesthetics and the underlying mechanisms under the controlled condition (e.g., anesthetic dose and exposure period).

\section{Acknowledgments}

This work has been supported in part by P01GM066730, R01HL034708 from the NIH, Bethesda, MD, and by FP00003109 from Advancing a Healthier Wisconsin Research and Education Initiative Fund (to ZJB).

\section{References}

1. Chalon J, Tang CK, Ramanathan S, Eisner M, Katz R, Turndorf H. Exposure to halothane and enflurane affects learning function of murine progeny. Anesth Analg. 1981; 60(11):794-7. [PubMed: 7197490]

2. Jevtovic-Todorovic V, Hartman RE, Izumi Y, Benshoff ND, Dikranian K, Zorumski CF, Olney JW, Wozniak DF. Early exposure to common anesthetic agents causes widespread neurodegeneration in the developing rat brain and persistent learning deficits. J Neurosci. 2003; 23(3):876-82. [PubMed: 12574416]

3. Mazoit JX, Roulleau P, Baujard C. Isoflurane-induced neuroapoptosis in the neonatal rhesus macaque brain: isoflurane or ischemia-reperfusion? Anesthesiology. 2010; 113(5):1245. author reply 1245-6. [PubMed: 20966672]

4. Slikker W Jr, Zou X, Hotchkiss CE, Divine RL, Sadovova N, Twaddle NC, Doerge DR, Scallet AC, Patterson TA, Hanig JP, Paule MG, Wang C. Ketamine-induced neuronal cell death in the perinatal rhesus monkey. Toxicol Sci. 2007; 98(1):145-58. [PubMed: 17426105]

5. Haley-Andrews S. Ketamine: the sedative of choice in a busy pediatric emergency department. J Emerg Nurs. 2006; 32(2):186-8. [PubMed: 16580488]

6. Young C, Jevtovic-Todorovic V, Qin YQ, Tenkova T, Wang H, Labruyere J, Olney JW. Potential of ketamine and midazolam, individually or in combination, to induce apoptotic neurodegeneration in the infant mouse brain. Br J Pharmacol. 2005; 146(2):189-97. [PubMed: 15997239]

7. Wilder RT, Flick RP, Sprung J, Katusic SK, Barbaresi WJ, Mickelson C, Gleich SJ, Schroeder DR, Weaver AL, Warner DO. Early exposure to anesthesia and learning disabilities in a populationbased birth cohort. Anesthesiology. 2009; 110(4):796-804. [PubMed: 19293700]

8. Kalkman CJ, Peelen L, Moons KG, Veenhuizen M, Bruens M, Sinnema G, de Jong TP. Behavior and development in children and age at the time of first anesthetic exposure. Anesthesiology. 2009; 110(4):805-12. [PubMed: 19293699]

9. Mellon RD, Simone AF, Rappaport BA. Use of anesthetic agents in neonates and young children. Anesth Analg. 2007; 104(3):509-20. [PubMed: 17312200]

10. Loepke AW, Soriano SG. An assessment of the effects of general anesthetics on developing brain structure and neurocognitive function. Anesth Analg. 2008; 106(6):1681-707. [PubMed: 18499597]

11. Thomson JA, Itskovitz-Eldor J, Shapiro SS, Waknitz MA, Swiergiel JJ, Marshall VS, Jones JM. Embryonic stem cell lines derived from human blastocysts. Science. 1998; 282(5391):1145-7. [PubMed: 9804556]

12. Shin S, Dalton S, Stice SL. Human motor neuron differentiation from human embryonic stem cells. Stem Cells Dev. 2005; 14(3):266-9. [PubMed: 15969621]

13. Vidarsson H, Hyllner J, Sartipy P. Differentiation of human embryonic stem cells to cardiomyocytes for in vitro and in vivo applications. Stem Cell Rev. 2010; 6(1):108-20. [PubMed: 20091143] 
14. Bissonnette CJ, Lyass L, Bhattacharyya BJ, Belmadani A, Miller RJ, Kessler JA. The controlled generation of functional Basal forebrain cholinergic neurons from human embryonic stem cells. Stem Cells. 2011; 29(5):802-11. [PubMed: 21381151]

15. Laposa R. Stem cells for Drug Screening. J Cardiovasc Pharmacol 2011. 2011; 27(12):3069-77.

16. Johnson MA, Weick JP, Pearce RA, Zhang SC. Functional neural development from human embryonic stem cells: accelerated synaptic activity via astrocyte coculture. J Neurosci. 2007; 27(12):3069-77. [PubMed: 17376968]

17. Nash R, Krishnamoorthy M, Jenkins A, Csete M. Human embryonic stem cell model of ethanolmediated early developmental toxicity. Exp Neurol. 2011; X:XX-X.

18. Domino EF, Zsigmond EK, Domino LE, Domino KE, Kothary SP, Domino SE. Plasma levels of ketamine and two of its metabolites in surgical patients using a gas chromatographic mass fragmentographic assay. Anesth Analg. 1982; 61(2):87-92. [PubMed: 7198883]

19. McLean RF, Baker AJ, Walker SE, Mazer CD, Wong BI, Harrington EM. Ketamine concentrations during cardiopulmonary bypass. Can J Anaesth. 1996; 43(6):580-4. [PubMed: 8773864]

20. Braun S, Gaza N, Werdehausen R, Hermanns H, Bauer I, Durieux ME, Hollmann MW, Stevens MF. Ketamine induces apoptosis via the mitochondrial pathway in human lymphocytes and neuronal cells. Br J Anaesth. 2010; 105(3):347-54. [PubMed: 20659914]

21. Campbell LL, Tyson JA, Stackpole EE, Hokenson KE, Sherrill H, McKeon JE, Kim SA, Edmands SD, Suarez C, Hall AC. Assessment of general anaesthetic cytotoxicity in murine cortical neurones in dissociated culture. Toxicology. 2011; 283(1):1-7. [PubMed: 21277931]

22. Mak YT, Lam WP, Lu L, Wong YW, Yew DT. The toxic effect of ketamine on SH-SY5Y neuroblastoma cell line and human neuron. Microsc Res Tech. 2010; 73(3):195-201. [PubMed: 19725066]

23. Ibla JC, Hayashi H, Bajic D, Soriano SG. Prolonged exposure to ketamine increases brain derived neurotrophic factor levels in developing rat brains. Curr Drug Saf. 2009; 4(1):11-6. [PubMed: 19149520]

24. Brambrink AM, Evers AS, Avidan MS, Farber NB, Smith DJ, Martin LD, Dissen GA, Creeley CE, Olney JW. Ketamine-induced neuroapoptosis in the fetal and neonatal rhesus macaque brain. Anesthesiology. 2012; 116(2):372-84. [PubMed: 22222480]

25. Hirokawa N, Sobue K, Kanda K, Harada A, Yorifuji H. The cytoskeletal architecture of the presynaptic terminal and molecular structure of synapsin 1. J Cell Biol. 1989; 108(1):111-26. [PubMed: 2536030]

26. Takadera T, Ishida A, Ohyashiki T. Ketamine-induced apoptosis in cultured rat cortical neurons. Toxicol Appl Pharmacol. 2006; 210(1-2):100-7. [PubMed: 16307766]

27. Dobbing J, Sands J. Comparative aspects of the brain growth spurt. Early Hum Dev. 1979; 3(1): 79-83. [PubMed: 118862]

28. Morrow BA, Roth RH, Redmond DE Jr, Sladek JR Jr, Elsworth JD. Apoptotic natural cell death in developing primate dopamine midbrain neurons occurs during a restricted period in the second trimester of gestation. Exp Neurol. 2007; 204(2):802-7. [PubMed: 17313945]

29. Samuelsen GB, Larsen KB, Bogdanovic N, Laursen H, Graem N, Larsen JF, Pakkenberg B. The changing number of cells in the human fetal forebrain and its subdivisions: a stereological analysis. Cereb Cortex. 2003; 13(2):115-22. [PubMed: 12507942]

30. Dekaban AS. Changes in brain weights during the span of human life: relation of brain weights to body heights and body weights. Ann Neurol. 1978; 4(4):345-56. [PubMed: 727739]

31. Zou X, Patterson TA, Sadovova N, Twaddle NC, Doerge DR, Zhang X, Fu X, Hanig JP, Paule MG, Slikker W, Wang C. Potential neurotoxicity of ketamine in the developing rat brain. Toxicol Sci. 2009; 108(1):149-58. [PubMed: 19126600]

32. Soriano SG, Liu Q, Li J, Liu JR, Han XH, Kanter JL, Bajic D, Ibla JC. Ketamine activates cell cycle signaling and apoptosis in the neonatal rat brain. Anesthesiology. 2010; 112(5):1155-63. [PubMed: 20418696]

33. Thompson CB. Apoptosis in the pathogenesis and treatment of disease. Science. 1995; 267(5203): 1456-62. [PubMed: 7878464] 
34. Crow MT, Mani K, Nam YJ, Kitsis RN. The mitochondrial death pathway and cardiac myocyte apoptosis. Circ Res. 2004; 95(10):957-70. [PubMed: 15539639]

35. Budd SL, Tenneti L, Lishnak T, Lipton SA. Mitochondrial and extramitochondrial apoptotic signaling pathways in cerebrocortical neurons. Proc Natl Acad Sci U S A. 2000; 97(11):6161-6. [PubMed: 10811898]

36. Zhang Y, Dong Y, Wu X, Lu Y, Xu Z, Knapp A, Yue Y, Xu T, Xie Z. The mitochondrial pathway of anesthetic isoflurane-induced apoptosis. J Biol Chem. 2010; 285(6):4025-37. [PubMed: 20007710]

37. Liu M, Liu H, Dudley SC Jr. Reactive oxygen species originating from mitochondria regulate the cardiac sodium channel. Circ Res. 2010; 107(8):967-74. [PubMed: 20724705]

38. de Grey AD. Antioxidants and redox signaling: Internet resources. Antioxid Redox Signal. 2000; 2(4):937-40. [PubMed: 11213493]

39. Himeda T, Kanbara S, Oki C, Kato H, Araki T. Effects of chronic administration with nilvadipine against immunohistochemical changes related to aging in the mouse hippocampus. Metab Brain Dis. 2005; 20(2):141-53. [PubMed: 15938132]

40. Perfettini JL, Roumier T, Kroemer G. Mitochondrial fusion and fission in the control of apoptosis. Trends Cell Biol. 2005; 15(4):179-83. [PubMed: 15817372]

41. Youle RJ, Karbowski M. Mitochondrial fission in apoptosis. Nat Rev Mol Cell Biol. 2005; 6(8): 657-63. [PubMed: 16025099]

42. Lee YJ, Jeong SY, Karbowski M, Smith CL, Youle RJ. Roles of the mammalian mitochondrial fission and fusion mediators Fis1, Drp1, and Opa1 in apoptosis. Mol Biol Cell. 2004; 15(11): 5001-11. [PubMed: 15356267]

43. Wu S, Zhou F, Zhang Z, Xing D. Mitochondrial oxidative stress causes mitochondrial fragmentation via differential modulation of mitochondrial fission-fusion proteins. FEBS J. 2011; 278(6):941-54. [PubMed: 21232014] 

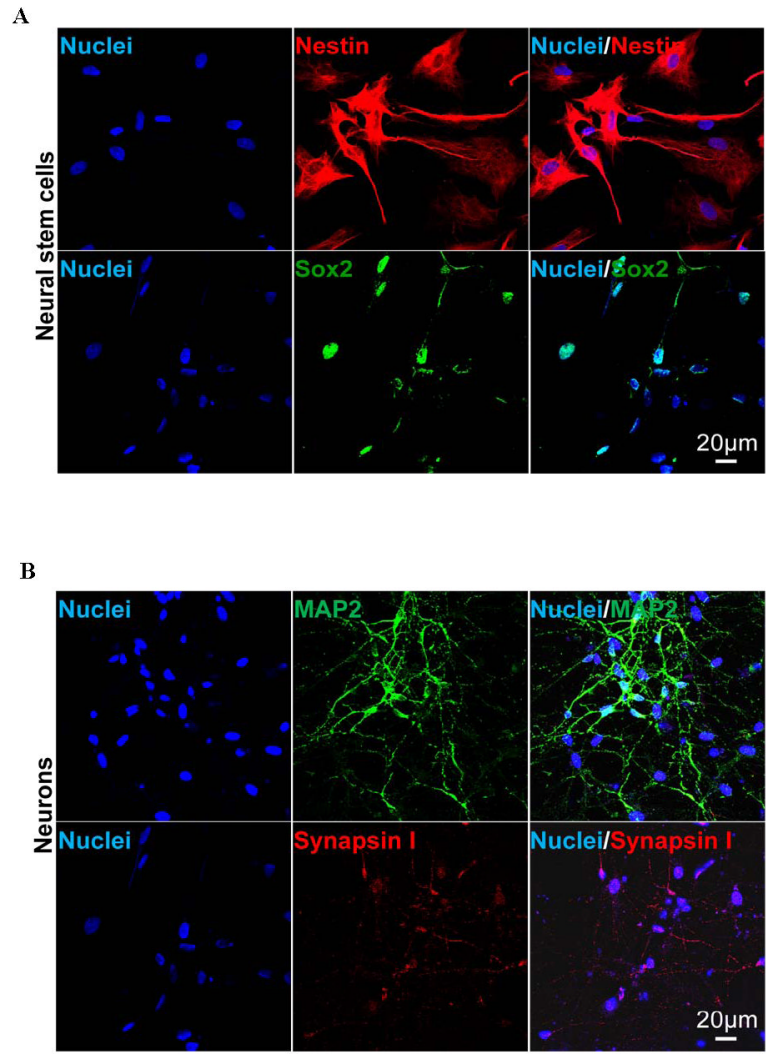

Figure 1. hESCs differentiate into neurons via neural stem cells (NSCs)

(A) Characterization of NSCs using immunofluorescence staining. Differentiated NSCs expressed NSC-specific marker nestin (upper panels, red) and Sox2 (bottom panels, green). Blue are cellular nuclei stained with TO-PRO®-3. (B) Neuronal characterization. Following two-week culture in neuronal differentiation medium, NSCs differentiated into neurons with small round cell body with extending long projections. Differentiated neurons were positive for neuron-specific marker MAP2 (upper panels, green) and synapse-specific marker synapsin 1 (bottom panels, red). Scale bar $=20 \mu \mathrm{m}$. 
A

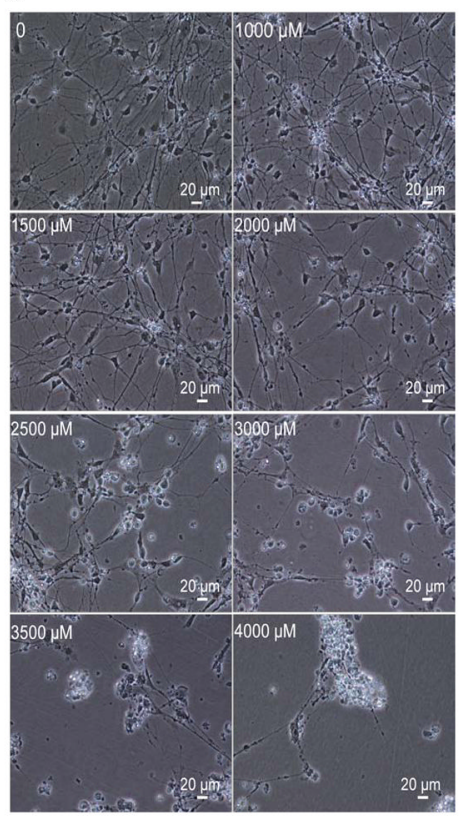

B

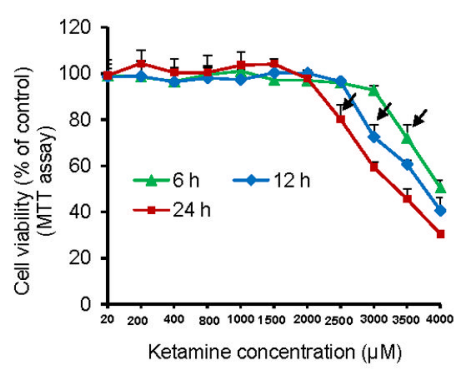

C

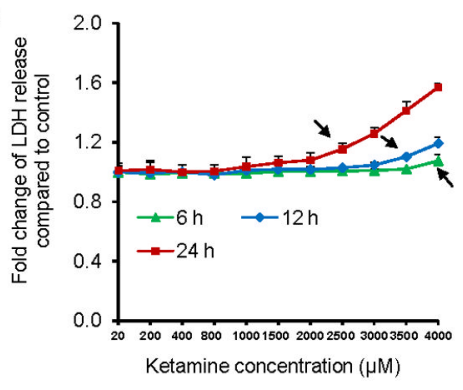

Figure 2. Ketamine induces cell death in a concentration- and time-dependent fashion (A) Representative phase contrast images of differentiated neurons treated with the indicated concentrations of ketamine for 24 hours. In response to $2500 \mu \mathrm{M}$ ketamine, neurons clearly began to retract their projections. In the $4000 \mu \mathrm{M}$ ketamine-contained culture, nearly all of the cells were detached. Scale bar is $20 \mu \mathrm{m}$. (B) MTT assay of mitochondrial dehydrogenase activity representing cell viability. Y-axis is the percentage of viable cells when compared with the control group after ketamine treatment. (C) LDH assay. Y-axis is the fold change in OD490 representing LDH release from the damaged cells. Higher dose and longer duration of exposure to ketamine resulted in greater cell death and LDH release. The arrows indicate at which point the difference are significantly different between ketamine treatment and control group $(\mathrm{P}<0.01, \mathrm{n}=3)$. 
A

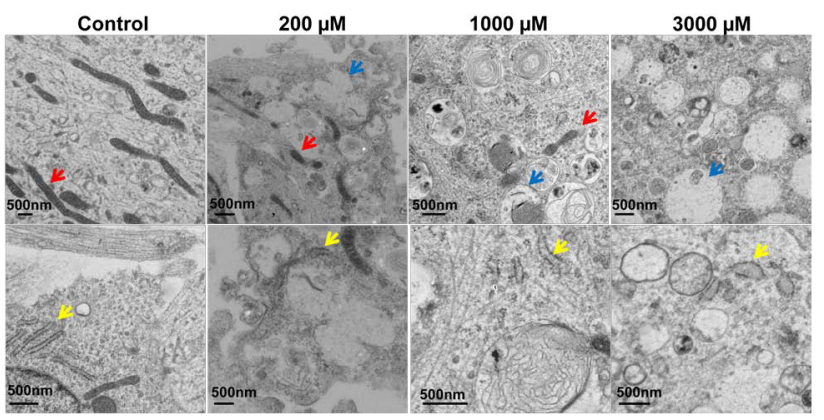

B

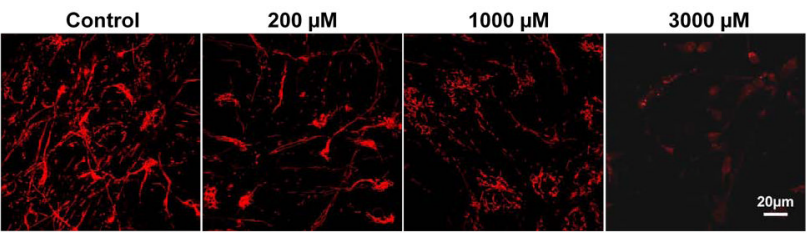

C

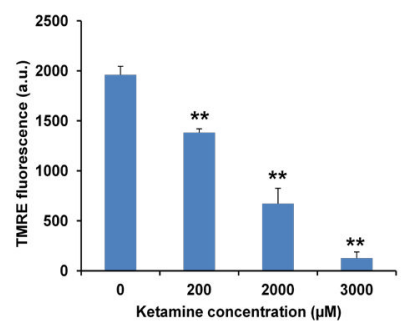

Figure 3. Ketamine at lower dosages induces abnormal cellular changes in ultrastructure and alteration in mitochondrial membrane potential $\left(\Delta \Psi_{m}\right)$ in a dose-depend manner

(A) Representative electron microscope images of differentiated neurons treated with the indicated concentrations of ketamine for 24 hours. Ketamine-treated neurons showed clear signs of the toxic effect on the cellular ultrastructure. Abnormal ultrastructure of neurons included fragmentation of mitochondria, decreased and distended rough ER, and many autophagosomes with or without being packed with dense amorphous material and other large clear vacuoles within the cytosol of all cells. Red, blue, and yellow arrows indicate mitochondria, autophagosome, and ER, respectively. Scale bars=500 nm. (B and C) Ketamine decreases $\Delta \Psi_{\mathrm{m}}$. Cells treated with ketamine for 24 hours were loaded with mitochondrial probe TMRE and imaged with the confocol microscope. Fluorescent intensity of TMRE represents $\Delta \Psi_{\mathrm{m}}$. The results show that ketamine treatment for 24 hours decreased $\Delta \Psi_{\mathrm{m}}$ in a dose-dependent fashion $(* * \mathrm{P}<0.01$, vs. control group, $\mathrm{n}=3)$. Scale bars $=20 \mu \mathrm{m}$. 
A

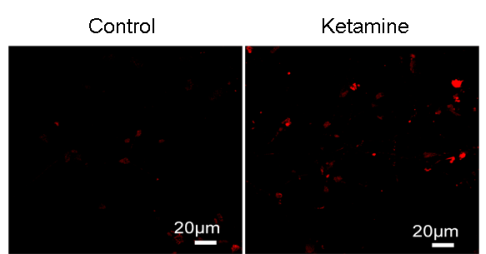

C

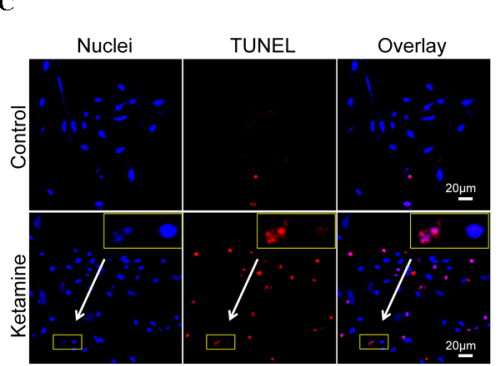

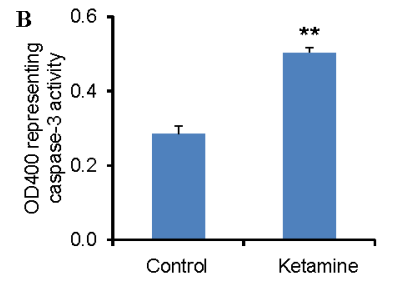

D

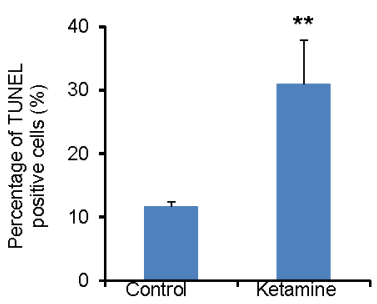

E

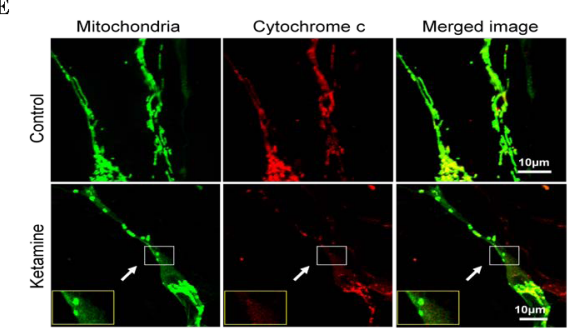

Figure 4. Ketamine stimulates active cleaved caspase-3- and TUNEL-positive apoptotic neurons, and induces the release of cytochrome $\mathbf{c}$ from mitochondria into cytosol

(A) Fluorescence images of active cleaved caspase-3 (red) in the neurons treated with 3000 $\mu \mathrm{M}$ ketamine for 24 hours analyzed using immunofluorescent staining. (B) Measurement of caspase- 3 activity in the lysate of neurons treated with or without $3000 \mu \mathrm{M}$ ketamine for 24 hours using Caspase-3 Colorimetric Assay Kits. (C) TUNEL staining. TUNEL staining (red) was used to identify cells containing DNA damage after $3000 \mu \mathrm{M}$ ketamine treatment for 24 hours. Cellular nuclei (blue) were stained with TO-PRO®-3. Overlaid images demonstrated that most of the TUNEL staining was located in the condensed or fragmented nuclei in ketamine-treated cells. The inset in the top right corner of each image is the magnified box indicated by arrow. Scale bars $=20 \mu \mathrm{m}$. (D) Quantification of TUNEL-positive cells. Statistically significant increases in caspase-3 activity and TUNEL-positive cells were observed in ketamine-treated cultures compared with untreated controls (B and D; $* * \mathrm{P}<0.01, \mathrm{n}=3$ ). (E) The distribution of cytochrome $\mathrm{c}$ within the neurons. In order to label mitochondria, neurons were treated with CellLight ${ }^{\mathrm{TM}}$ mitochondria-GFP reagent. The distribution of cytotochrome $\mathrm{c}$ in cells was analyzed by immunofluorescence staining. Column 1 is the image of mitochondria (green); column 2 is the image of cytochrome c (red); and column 3 is the merged image. The orange color in the merged images indicates the existence of cytochrome $\mathrm{c}$ inside the mitochondria and the red signals in the merged images indicate the existence of cytochrome $\mathrm{c}$ outside the mitochondria. The inset in the bottom left corner of each image is the magnified box indicated by arrow. The results show that $3000 \mu \mathrm{M}$ ketamine treatment for 24 hours resulted in the cytochrome c release from the mitochondria into cytosol as compared with the control condition. 


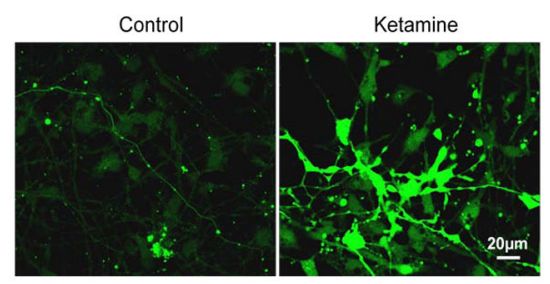

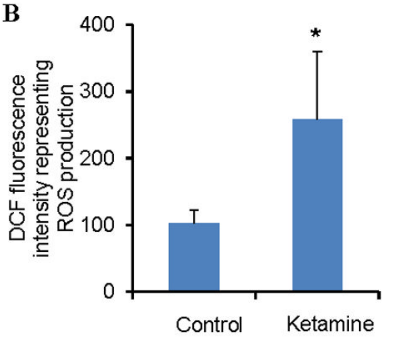

C

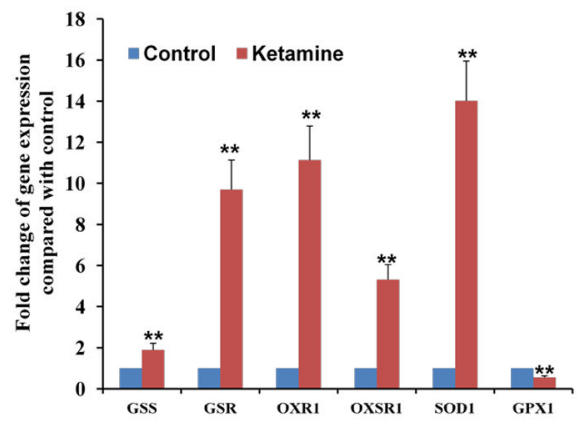

Figure 5. Ketamine increases reactive oxygen species (ROS) production and induces differential regulation of oxidative stress-related gene expression in neurons

(A) Confocol images of ROS production of neurons in the control and $3000 \mu \mathrm{M}$ ketaminetreated culture for 24 hours. Neurons were loaded with oxidative fluorescent indicator dye $\mathrm{CM}-\mathrm{H}_{2}$ DCFDA and the produced ROS (green) is observed in the cells. Scale bar=20 $\mu \mathrm{m}$. (B) Quantification of ROS production in neurons. ROS level was significantly higher in the ketamine-treated cells than in the no-treatment culture $(* \mathrm{P}<0.05, \mathrm{n}=3)$. $(\mathbf{C})$ Real-time PCR analysis of fold changes of oxidative stress-related gene expression in neurons following $3000 \mu \mathrm{M}$ ketamine exposure for 24 hours compared with no-treatment control. $\mathrm{n}=3$. Glutathione synthetase (GSS), glutathione reductase (GSR), oxidation resistance 1 (OXR1), oxidative-stress responsive 1 (OXSR1), superoxide dismutase 1 (SOD1), and glutathione peroxidase 1 (GPX1). 
A

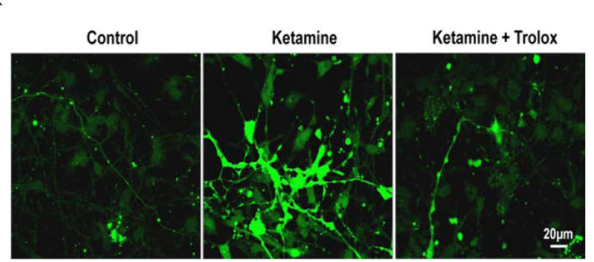

oum.

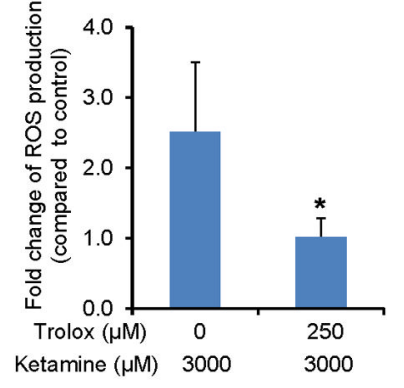

C

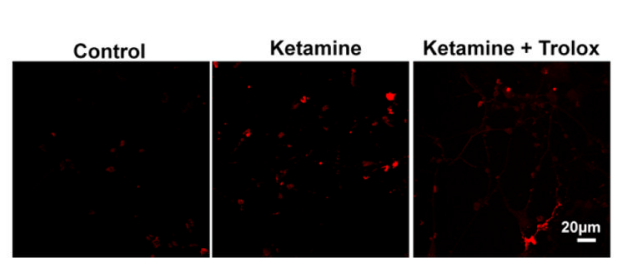

E

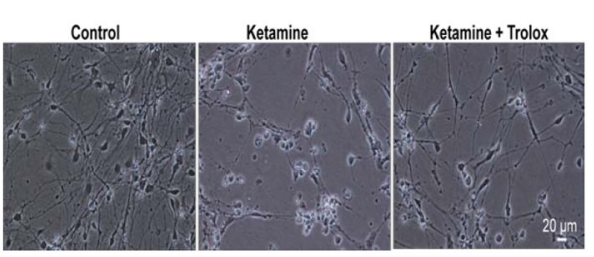

D

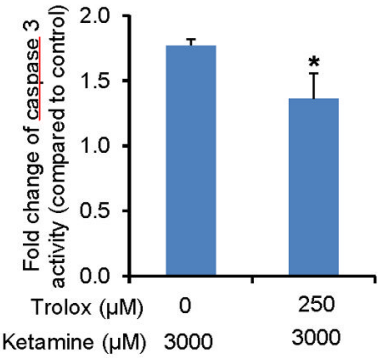

F

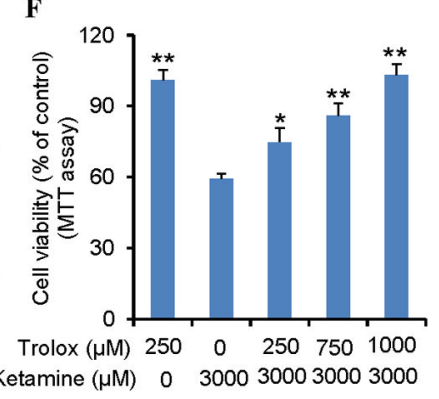

Figure 6. ROS inhibitor Trolox attenuates ketamine-induced neurotoxicity

Neurons were treated with $3000 \mu \mathrm{M}$ ketamine in the presence or absence of Trolox for 24 hours and then subjected to ROS measurement, caspase- 3 activity analysis and MTT assay. (A) Confocol images of ROS production of neurons in the cultures with or without ketamine and Trolox $(250 \mu \mathrm{M})$ treatment. (B) Trolox significantly decreased ketamine-induced ROS production. (C) Fluorescence images of active cleaved caspase-3 (red) in the neurons treated with or without ketamine and Trolox $(250 \mu \mathrm{M})$. (D) Trolox decreased cleaved caspase 3 activity of neurons following ketamine exposure. (E) Phase contrast images of differentiated neurons treated with or without ketamine and Trolox $(250 \mu \mathrm{M})$. (F) Trolox attenuated the ketamine-induced decrease in cell viability in a dose-dependent manner $(* \mathrm{P}<0.05$, $* * \mathrm{P}<0.01$, vs. ketamine alone group, $\mathrm{n}=3$ ). Scale bars are $20 \mu \mathrm{m}$. 